\title{
Soil Burial, Hygrothermal and Morphology of Durian Skin Fiber Filled Polylactic Acid Biocomposites
}

\author{
Ming Meng Pang1, Yesudian Aaron'1, Seong Chun Koay', Jiun Hor Low ${ }^{1}$, Hui Leng Choo', Kim Yeow Tshai ${ }^{2}$
}

${ }^{1}$ School of Engineering, Taylor's University, Lakeside Campus, No.1, Jalan Taylor's, 47500 Subang Jaya, Selangor, Malaysia.

${ }^{2}$ Faculty of Engineering, University of Nottingham Malaysia Campus, Jalan Broga, 43500 Semenyih, Selangor, Malaysia.

Correspondence Author: M.M. Pang, Taylor's University, Chemical Engineering Department, School of Engineering, No.1, Jalan Taylor's, 47500 Subang Jaya, Selangor, Malaysia. Tel: 60356295414

Email: mingmeng.pang@taylors.edu.my, pangmingmeng@hotmail.com

Received date: 12 January 2018, Accepted date: 28 February 2019, Online date: 20 March 2019

Copyright: (C) 2019 Ming M. Pang et al., This is an open-access article distributed under the terms of the Creative Commons Attribution License, which permits unrestricted use, distribution, and reproduction in any medium, provided the original author and source are credited.

\begin{abstract}
In this work, the alkali ( $\mathrm{NaOH}$ ) treated and untreated durian skin fibers (DSF) were added into polylactic acid (PLA) to form biocomposites. The PLA/DSF biocomposite preparation was performed via melt blending method $\left(180^{\circ} \mathrm{C}, 50 \mathrm{rpm}\right.$ and 10 mins retention time) at different loadings of DSF, i.e., 15, 25, 30 and 40 wt\%, followed by compression molding to form thin sheet. The experimental investigation was carried out to study the weight loss of the PLA/DSF subjected to soil burial for 80 days and hygrothermal test for 14 days. Results showed that the incorporation of DSF can accelerate the weight loss of PLA, higher weight loss was observed at higher fiber content in both soil burial and hygrothermal test. Scanning electron microscope (SEM) micrographs showed the presence of crack line and voids, signs of degradation for soil buried sample.
\end{abstract}

Keywords: Soil burial, hygrothermal, durian skin fiber, polylactic acid

\section{INTRODUCTION}

Durian husk or skin is the waste product after the consumption of the durian fruit, which is known by local as the "King of fruits" [1]. The disposal of durian husk especially in landfill has caused various environmental issues such as soil contamination and disease spreading. The utilization of the natural fiber obtained from durian husk is seen as a potential solution to reduce the waste disposal and to diversify the usage of agricultural waste.

The natural fiber is more eco-friendly and is seen as a suitable replacement for synthetic fiber. The natural fiber is lightweight, non-abrasive, renewability and biodegradability, which found applications in many fields ranging from a consumer product to the automotive industry. The examples of natural fibers are fruit fibers, wood, silk, ramie, jute, hemp, kenaf, sisal, coir, flax and bamboo [2]. The natural fiber obtained from agricultural waste such as durian skin fiber (DSF) is inexpensive and can be used as a reinforcement agent in a polymer matrix to form biocomposite. Biocomposite can be referred to a multi-phase material in which reinforcement fillers are added and integrated into a polymer matrix, resulting in synergistic properties that cannot be achieved from either component alone [3].

Polylactic acid (PLA) is one of the most common biopolymers which is derived from a renewable resource. PLA has attracted much attention due to its advantages such as high strength, high modulus, compostable and regarded as a safe material for food packaging application [4]. However, the disadvantages of the PLA are having low thermal stability and low elongation property. Previous works have been carried out to improve the performance of the PLA by adding reinforcement agent or filler to form biocomposites. Sun et al. [5] reported the good interfacial adhesion between the PLA and treated coir fiber was attributed to the pretreatment of fiber, which led to the improvement in tensile modulus and impact strength. According to Koay et al. [6], the addition of untreated DSF increased the tensile strength and modulus of the recycled polystyrene foam/DSF composites but decreased the elongation at break. The good interfacial adhesion between the natural fibers and the matrix is important for the superior properties, and this can be achieved via physical or chemical methods. Physical methods include plasma and heat treatment of natural fiber. Meanwhile, chemical methods include alkaline treatment, acetylation and the use of coupling agent [7].

In this study, DSF was obtained from durian husk and treated with an alkaline solution (sodium hydroxide) to remove lignin, hemicellulose, wax, and oil covering the external surface of the fiber cell wall [8]. The chemical composition of the DSF was 
reported to consist of $68.1 \%$ cellulose, $16.2 \%$ hemicellulose, $13.7 \%$ lignin, and $0.5 \%$ ash [9]. There is a lack of research works or publications on the treated DSF filled PLA biocomposite. Hence, this article investigated the effect of the treated DSF loadings on PLA and evaluated the weight loss of the PLA/DSF biocomposites subjected to soil burial and hygrothermal test for 80 and 14 days, respectively.

\section{Materials:}

\section{METHODOLOGY:}

The PLA pellet (grade 3051D) was purchased from Nature Work LLC, and the durian skin was obtained from the local stall at no cost. Sodium hydroxide $(\mathrm{NaOH})$ was purchased from R\&M Chemicals.

\section{Method: \\ Sample preparation:}

The durian skin was first washed with tap water, sun-dried and cut into smaller pieces. The smaller pieces of durian skin were oven dried overnight at $70^{\circ} \mathrm{C}$. The dried durian skin was then grinded into short fiber named DSF, sieved with a mesh size of 600 micron to obtain the homogenous size of untreated DSF. The next step was to prepare the treated DSF by soaking in 5\% of $\mathrm{NaOH}$ solution for an hour. The treated DSF were washed thoroughly with distilled water to remove the $\mathrm{NaOH}$ residue and dried again in the oven at $70^{\circ} \mathrm{C}$ for overnight. The purpose of the pre-treatment with $\mathrm{NaOH}$ was to enhance the adhesion between the natural fiber and polymer matrix. The PLA/DSF biocomposites were prepared via melt blending method (using Haake Rheomix at $180^{\circ} \mathrm{C}$, rotor speed of $50 \mathrm{rpm}$ and retention time of $10 \mathrm{~min}$ ) according to the formulation listed in Table 1 . The amount of treated DSF was added at the range of $0,15,25$ to $40 \mathrm{wt} \%$ and designated as PLA, $15 \%$ treated DSF, $25 \%$ treated DSF, and $40 \%$ treated DSF. There was one formulation designated as $30 \%$ untreated DSF to indicate the addition of $30 \mathrm{wt} \%$ of untreated DSF into the PLA matrix. Compression machine (model: Moore E53) was used to mould the composites into a rectangular thin sheet of 1 mm thickness. The moulding steps included pre-heating at $180^{\circ} \mathrm{C}$ for 5 mins, followed by fully compression process for 2 min with $150 \mathrm{bar}$ and last, cooled the thin sheet for $8 \mathrm{~min}$. The sheets obtained from compression moulding were cut into a square specimen of $2 \mathrm{~cm} \times 2 \mathrm{~cm}$ for soil burial and hygrothermal testing.

Table 1. Formulation for biocomposite

\begin{tabular}{ccc}
\hline Sample & PLA $(\mathrm{wt} \%)$ & DSF $(\mathrm{wt} \%)$ \\
\hline PLA & 100 & 0 \\
15\% treated DSF & 85 & 15 \\
25\% treated DSF & 75 & 25 \\
40\% treated DSF & 60 & 40 \\
$30 \%$ untreated DSF & 70 & 30 \\
\hline
\end{tabular}

\section{Characterization: \\ Soil burial test:}

The initial weight of each specimen was recorded before buried in a gardening pot at a depth of $10 \mathrm{~cm}$ from the surface. The specimens were subjected to oven drying before and after soil burial at $50^{\circ} \mathrm{C}$ for $24 \mathrm{hr}$. The gardening soil with $\mathrm{pH} 6.9 \mathrm{was}$ used. The soil was kept moist by spraying water regularly to promote microbial activity. The specimens were removed from the soil periodically to monitor the weight loss, and the soil burial test last for 80 days. Specimens were cleaned, dried and kept in a desiccator before weighing. The average weight of the specimens was obtained to calculate the percentage of weight loss by using Eq. 1.

$$
\text { Percentage of weight loss }(\%)=\frac{W_{0}-W_{t}}{W_{0}} \times 100 \% \quad \text { Eq. } 1
$$

where $W_{0}$ is the initial weight and $W_{t}$ is the final weight of the specimen in gram.

\section{Hygrothermal test:}

The specimen was immersed into a water bath filled with distilled water at a temperature of $58^{\circ} \mathrm{C}$. Before immersion, the initial weight of each specimen was recorded. This hygrothermal test investigated the weight change in the PLA/DSF biocomposites after exposure to a combination of heat and moisture attack. The specimen was removed from the water bath at a different time interval and wiped with tissue to measure the final weight. The weight loss calculation is the same as Eq.1.

\section{Scanning electron microscope (SEM):}

The surface morphology of the PLA/DSF specimen before and after soil burial test was examined by using the FEI Quanta $400 \mathrm{~F}$ FESEM at an accelerating voltage of $10 \mathrm{kV}$.

\section{Soil burial test:}

\section{RESULTS AND DISCUSSION}

A soil burial test was performed on a laboratory scale to investigate the biodegradability of the PLA/DSF composites. Figure 1 shows the weight loss $(\%)$ of the soil buried specimens. The $30 \%$ untreated DSF reported the highest percentage weight loss for all the days the weights were measured. Meanwhile, pure PLA reported the least percentage weight loss over all the other samples 
being tested. Overall can see as the duration of buried time increased, the greater weight loss was observed, except on the day-45 and day-70 where there were fluctuations of data. The biocomposites with treated DSF loadings of 15,25 and $40 \%$ showed an average percentage weight loss between 2 to $3 \%$ and the higher the DSF content, the greater the weight loss recorded.

The 30\% untreated DSF biodegraded faster in the soil as compared to treated DSF biocomposites, with the weight loss of 5.6\% at day-80. The untreated DSF contained a higher amount of amorphous materials such as lignin and hemicellulose, the disordered nature of amorphous region made it more susceptible for the microbial attack [10,11]. Moreover, the alkali pre-treatment step increased the interfacial adhesion between the fiber and the matrix [12], which gave resistance to the treated DSF composites neither hydrolysis nor microbial attack.

PLA showed the negligible weight loss of $\sim 1 \%$ at day- 80 . This result was in accordance with the previous studies that reported slow degradation of PLA in soil, attributed to the low hydrolysis rate at low temperature and water content, in addition, PLAdegrading microorganisms are not widely found in the natural environment which makes the PLA is less susceptible to microbial attack in a natural environment as compared to other aliphatic polyesters [13, 14].

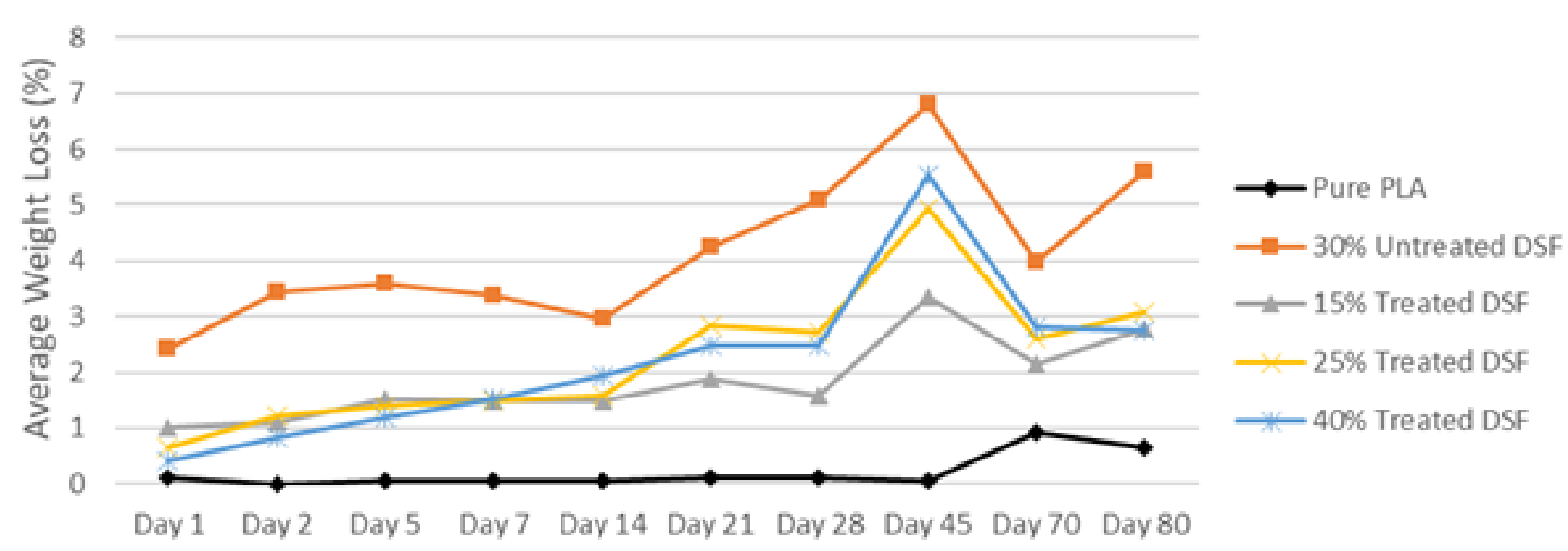

Figure 1. Soil burial weight loss trend over 80 days.

\section{Hygrothermal test;}

The hygrothermal test was performed to understand the durability of the PLA/DSF biocomposites after exposure to heat and moisture for 14 days. Figure 2 shows the weight loss of the PLA/DSF compositesincreased with time. As being fully immersed in the water bath at a temperature of $58^{\circ} \mathrm{C}$, the specimens were expected to gain weight at beginning stage due to the water absorption until reached a saturation point (this happened rapid and not reflected in figure 2), then experienced hygrothermal degradation beyond the saturation point. The hygrothermal degradation is an aging process which combines the impacts of both moisture and temperatures, resulting in the substantial weight loss and deterioration of mechanical properties [15].

The weight loss in the PLA/DSF biocomposites was mainly caused by the dissolution of DSF from the developed micro-crack owing tothe hygrothermal environment. The PLA matrix experienced hydrolytic degradation under the moisture and heat attack via random cleavage of ester bond; this causes the long polymer chain to break into shorter water-soluble fragments [16]. In this study, the $30 \%$ untreated DSF showed the highest weight loss for the first seven days at $9 \%$. Subsequently the weight loss was gradually slow down at day-14. The highest weight loss in untreated DSF biocomposite was attributed to the dissolution of the amorphous materials such as lignin andhemicellulose. The alkali treatment removed most of the amorphous materials and left with cellulose in DSF. Cellulose is composed of crystalline and amorphous regions [11]. The amorphous region can easily penetrate by chemicals or water, whereas the compactness of the crystalline region makes it hard for chemical penetration. Among the treated DSF specimens, the weight loss increases withan increase in the fiber content. The $40 \%$ treated DSF recorded the highest weight loss at $17 \%$ while $15 \%$ treated DSF recorded weight loss at $14 \%$ at day-14. The pure PLA was resistance to the hygrothermal degradation for the first 7 days and only encountered $2.2 \%$ weight loss by day-14 due to the hydrolysis of PLA. 


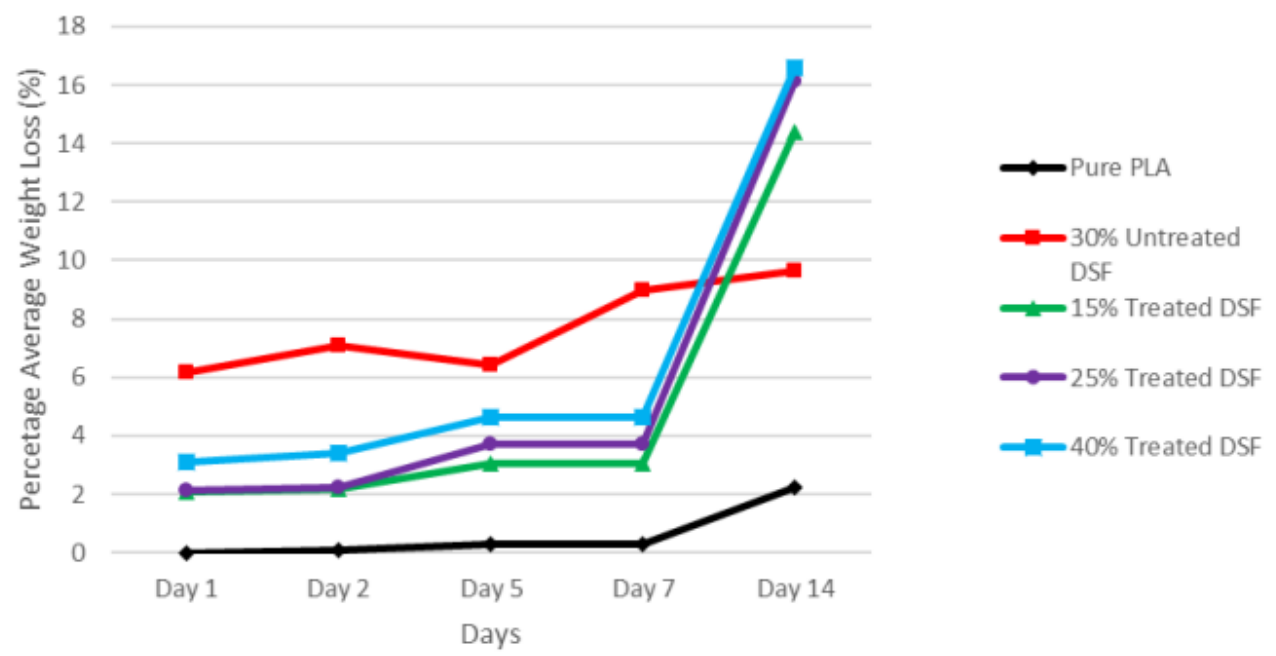

Figure 2. Hygrothermal weight loss trend over 14 days.

\section{Morphology:}

The surface morphology for the $30 \%$ untreated DSF biocomposite before and after soil burial for 60 days is shown in Figure 3. Before soil burial (Figure 3a), the specimen surface was smooth with no crack line, however, after soil burial (Figure 3b), pores and crack lines can be found on the surface of the biocomposite. Similar findings reported on the $40 \%$ treated DSF biocomposite (Figure 4), the specimen surface was smooth before soil burial, and crack lines were seen after soil burial for 60 days. The bigger size of cracks and pores were observed on the $30 \%$ untreated DSF, and this could explain the highest weight loss encountered by the untreated DSF as compared to treated DSF biocomposites during soil burial test.
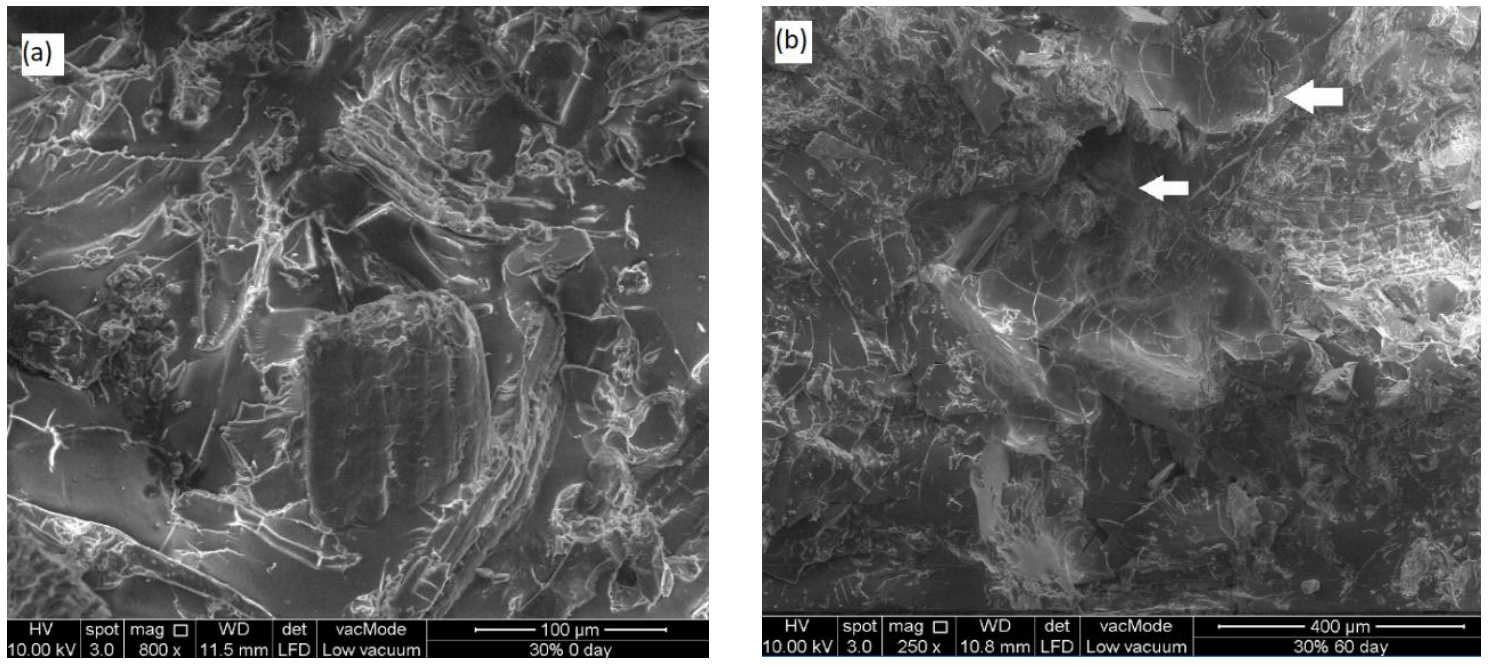

Figure 3. SEM micrographs for $30 \%$ untreated DSF (a) before (b) after soil burial for 60 days.
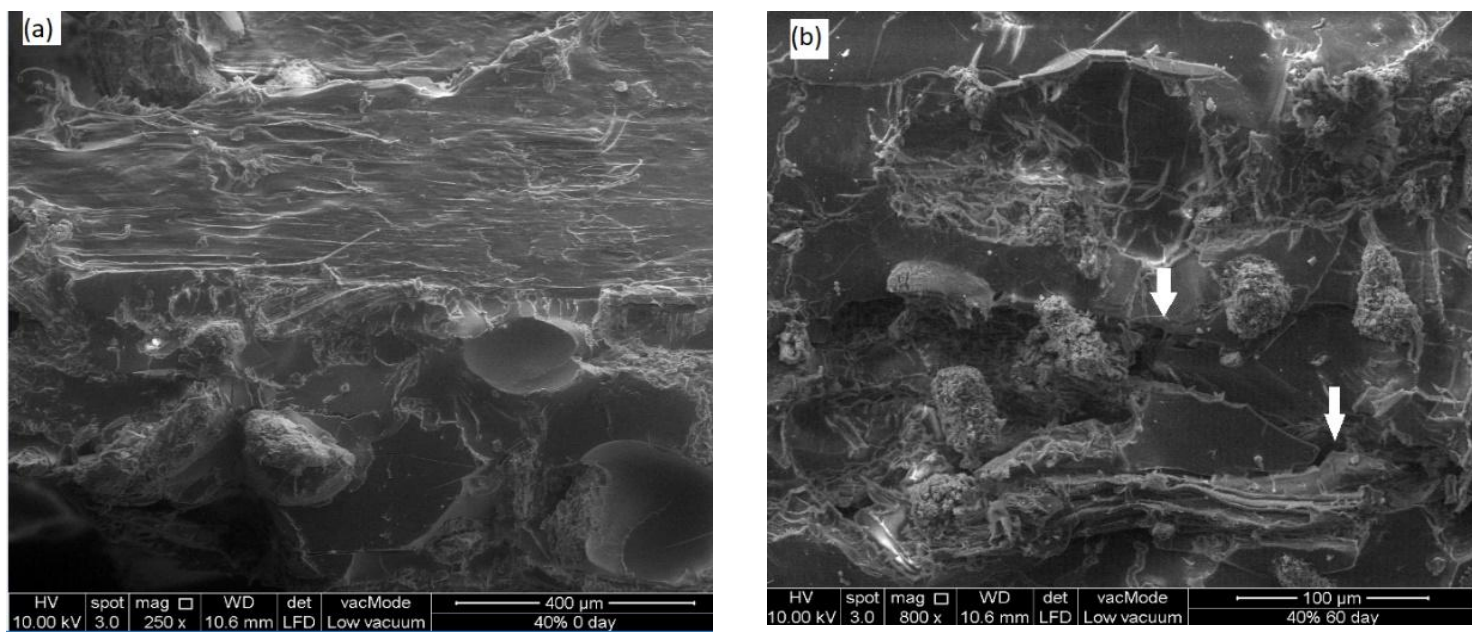
Figure 4. SEM micrographs for $40 \%$ treated DSF (a) before (b) after soil burial for 60 days.

\section{CONCLUSIONS}

This work examined the degradation (weight loss) of the untreated and treated DSF filled PLA biocomposites after exposure to soil burial and hygrothermal tests. The incorporation of DSF as lignocellulosic material can accelerate the biodegradability of the PLA in soil. The untreated DSF showed higher weight loss than treated DSF, as the latter contained lesser amorphous materials such as hemicellulose and lignin after alkaline treatment, and the amorphous fraction of the biocomposites was more susceptible for microbial attack. In the hygrothermal test, the untreated DSF again showed higher weight loss due to a higher fraction of the amorphous region, which made it more susceptible to moisture and heat attack. Overall, the degradation (weight loss) of PLA/DSF biocomposites increased with the increasing DSF content.

\section{ACKNOWLEDGEMENTS}

This research was financially supported by Taylor's University, Malaysia (Grant: TRGS/ERFS/1/2017/SOE/031).

\section{REFERENCES}

1. Manshor, R.M., H. Anuar, W.B. Wan Nazri, and M.I. Ahmad Fitrie, 2012. Preparation and characterization of physical properties of durian skin fibers biocomposite. Advanced Materials Research, 576:212- 215.

2. Ranakoti, L., M. Pokhriyal and A. Kumar, 2018. Natural fibers and biopolymers characterization: a future potential composite material. Journal of Mechanical Engineering, 68(1): 33 - 50.

3. Wang, G., D. Yu, A.D. Kelkar and L. Zhang, 2017. Electrospun nanofiber: emerging reinforcing filler in polymer matrix composite materials. Progress in Polymer Science, 75: 73-107.

4. Jasim, A. and K.V. Sunil, 2011. Polylactides-Chemistry, properties and green packaging technology: a review. Internation Journal of Food Properties, 14: 37-58.

5. Sun, Z., L. Zhang, D., Liang, W. Xiao and J. Lin, 2017. Mechanical and thermal properties of PLA biocomposies reinforced by coir fibers. International Journal of Polymer Science, 2178329.

6. Koay, S.C., V. Subramanian, M.Y. Chan, M.M. Pang, K.Y. Tsai and K.H. Cheah, 2018. Preparation and characterization of wood plastic composite made up of durian husk fiber and recycled polystyrene foam. MATEC Web Conf 152, 02019.

7. Li, K., R. Qiu and W. Liu, 2015. Adhesion in natural plant fiber-reinforced unsaturated polyester composites: a critical review. Reviews of Adhesion and Adhesives, 3(1):98-120.

8. Li, X., L.G. Tabil and S. Panigrahi, 2007. Chemical treatments of natural fiber for use in natural fiber-reinforced composites: a review. Journal of Polymers and the Environment, 15: 25-33.

9. Nur Aimi, M.N., H. Anuar, M. Maizirwan, S.M. Sapuan, M.U. Wahit and S. Zakaria, 2015. Preparation of durian skin nanofibre (DSNF) and its effect on the properties of polylactic acid (PLA) biocomposites. Sains Malaysiana, 44(11):1551 1559.

10. Mu, B., H. Wang, X.Hao and Q. Wang, 2018. Morphology, mechanical properties and dimensional stability of biomass particles/high density polyethylene composites: effect of species and composition. Polymers, 10(3), 308.

11. Nasution, H., Yurnaliza, Veronicha, Irmadani and S. Sitompul, 2017. Preparation and characterization fo cellulose microcrystalline (MCC) from fiber of empty fruit bunch palm oil. IOP Conferences Series: Materials Science and Engineering, 180, 012007.

12. Yu, T., J. Ren, S. Li, H. Yuan and Y. Li, 2010. Effect of fiber surface-treatments on the properties of poly(lactic acid)/ramie composites. Composites Part A, 41: 499-505

13. Vasile, C., D. Pamfil, M. Râpă, R.N. Darie-Niţă, A.C. Mitelut, E.E. Popa, P.A. Popescu, M.C. Draghici and M.E. Popa, 2018. Study of the soil burial degradation of some PLA/CS biocomposites. Composites Part B, 142: 251-262.

14. Qi, X., Y. Ren and X. Wang, 2017. New advances in the biodegradaton of poly(lactic) acid. International Biodeterioration \& Biodegradation, 117: 214-223.

15. Chow, W.S., A. Abu Bakar and Z.A. Mohd Ishak, 2005. Water absorption and hygrothermal aging study on organomontmorillonite reinforced polyamide 6/polypropylene nanocomposites. Journal of Applied Polymer Science, 98: 780-790.

16. Muthuraj, R., M. Mistra and A.K. Mohanty, 2015. Hydrolytic degradation of biodegradable polyesters under simulated environmental conditions. Journal of Applied Polymer Science, 132(27): 42189-42201. 